\title{
Journal of Applied Mechanical Engineering
}

\section{Fourier Analysis of Milling Force for General Helical Cutters via Space- time Convolution, Part 2: Applications for Common Cutters and Model Validation}

\author{
Zheng $\mathrm{CM}^{1 *}$ and Junz Wang $\mathrm{JJ}^{2}$ \\ ${ }^{1}$ Department of Mechanical and Electrical Engineering, Quanzhou Institute of Information Engineering, Quanzhou 36200, PR China \\ ${ }^{2}$ Department of Mechanical Engineering, National Cheng Kung University Tainan, 701, Taiwan
}

\begin{abstract}
Part 2 of this study illustrates the applications of the frequency domain force model put forth in Part 1 for three common helical cutters: the square, taper, and ball end mills. The respective geometric and boundary functions required for the evaluation of the force spectra are derived by applying differential geometry to these three types of cutters including cutters of constant helix angle and constant helix lead. By virtue of the explict expression of Fourier coefficients of milling force, the differences between cutting forces generated by two cutters with constant helix angle and constant helix lead can be described quantitatively. In slot (or half slot) milling for the taper end mills with a constant helix angle and constant helix lead, the strategy for selecting axial depths of cut to reduce force pulsation is presented respectively. Also derived are the specific expressions for the average forces of these three helical cutters in common cutting configurations. Moreover, as an inverse application, a linear equation is formulated for the identification of six shearing and ploughing cutting constants from the measured average cutting forces for a general helical cutter. The frequency domain force model and the identification of the cutting constants are finally demonstrated and validated through experiments with all three types of milling cutters.
\end{abstract}

Keywords: Ball-end milling; Taper-end milling; General helical mill; Differential geometry

\section{Introduction}

The convolution force model and the force spectra characteristics of a general helical milling cutter has been presented and analyzed in Part 1 of this paper. It has been shown that the composing structures of the milling force in the angular and the frequency domain are the same for different types of helical cutters. Also the frequency spectra of the milling forces are shown to be characterized by the geometric functions of the helical cutting edge and by the cutting boundary functions which are determined by the axial and radial cutting depths. Therefore, the task of establishing the specific milling force models for any type of helical cutter is reduced to finding the analytic expressions for the line geometry of its helical cutting edge, as well as the expressions for the cutting boundaries including the entry and exit angle and the limits of the radial angle immersion. Although this generalized analytical model is applicable for any cutter with an analytically definable cutting edge, the second part of this paper will only illustrate its application for three common industrial helical end mills: the square, taper, and ball end mills.

Kinematics and cutting forces for the square end mill dates back to Martellotti $[1,2]$. However, force models for cutters other than the face milling cutter and square end mill only began in the 1990's. Yang and Park [3] could have been the first to present a force model for the ball-end mill. In their work, the force model is based on the fundamental mechanics of orthogonal cutting and explicitly considers the effects of the shear angle, shear stress and friction angle. Other researchers [4-7] have studied the force model for the ball end mill with a mechanistic local force model, in which cutting constants are assumed to be proportional to the uncut chip area and are obtained through milling experiments. Unlike the mechanistic local force model, Sonawane and Joshi [8] presented a analytical force model for the ball-end milling of superalloy Inconel 718 considering strain, strain rate, and temperature dependence of work material shear strength by applying Johnson-Cook material mode. The model analysis shows that there is a significant compression in the chip along its length. The taper end mill is not as common as the square or ball end mills in its industrial uses and has received less attention in the study of its force model. Ramaraj and Eleftheriou [9] used oblique cutting theory in establishing the force model for a taper end mill. Based on the mechanistic local force model, Huang and Whitehouse [10] obtained the total forces through numerical integration for a taper end mill. Instead of dealing with a helical cutter of specific geometry, Altintas et al. [11,12] presented force and process models for a general helical cutter through numerical integration and simulation. With emphasis on the composing structure of the milling forces, the present frequency domain force model is established using a systematic bottom-up approach through the principles of differential geometry, and can be de-generalized to accommodate any helical cutter of definite geometry. In the following, the required geometric and cutting boundary functions will be derived for the cylindrical, taper and ball end mill to complete the force model for each respective cutter. Expressions for their average forces are also derived and subsequently used for the identification of shearing and ploughing constants for each cutter. The dual-mechanism frequency domain force model and the cutting constant identification formula are finally validated through milling experiments with all three types of cutters.

*Corresponding author: Zheng CM, Department of Mechanical and Electrical Engineering, Quanzhou Institute of Information Engineering, Quanzhou 36200, PR China, Tel: 1-86-595-22765; E-mail: cmzheng1206@gmail.com

Received December 21, 2015; Accepted January 21, 2016; Published January 23, 2016

Citation: Zheng CM, Junz Wang JJ (2016) Fourier Analysis of Milling Force for General Helical Cutters via Space-time Convolution, Part 2: Applications for Common Cutters and Model Validation. J Appl Mech Eng 5: 195. doi:10.4172/21689873.1000195

Copyright: (C) 2016 Zheng CM, et al. This is an open-access article distributed under the terms of the Creative Commons Attribution License, which permits unrestricted use, distribution, and reproduction in any medium, provided the original author and source are credited. 


\section{Geometric Functions and Cutting Boundary Functions for Common Helical Cutters}

The evaluation of the Fourier coefficients for a specific cutter requires its geometric functions in $\psi(\beta)$ and $h^{\prime}(\beta)$, as well as the cutting boundary functions in $\beta 1, \beta 2, \theta 1(\beta)$ and $\theta 2(\beta)$. Although the geometric functions can be completely determined for any mathematically definable cutter, the boundary functions depend not only on the cutter geometry but also on the axial and radial depths of cut and the relative position of the work with respect to the cutter. As illustrative examples, the boundary conditions are derived based on the typical cutting configurations shown in Figure 1.

\section{Square end mills}

For the square end mill shown in Figure 1a, it is clear that the cutter radius is a constant $\mathrm{R}$ and the axial elevation angle is also a constant with $\psi=90^{\circ}$. It is assumed that the helix angle is constant and so is the helix lead,

$$
h^{\prime}(\beta)=\frac{R}{\tan \alpha} \text { and } h(\beta)=\frac{R}{\tan \alpha} \beta
$$

From (1), the end points of the radial cutting range are found to be $\beta 1=0$ and $\beta 2=d a \tan \alpha / R$. With a constant radial depth of cut and radius, the entry/exit angles are also constants, which are

$$
\begin{aligned}
& \theta_{1}=0 \text { and } \theta_{2}=\cos ^{-1}\left(1-\frac{d r}{R}\right) \text { in up milling and } \\
& \theta_{1}=\cos ^{-1}\left(1-\frac{d r}{R}\right) \text { and } \theta_{2}=180 \text { in down milling }
\end{aligned}
$$

Thus, for square end mill, the geometric and boundary functions all have constant values. Substituting these constants, the expression for the Fourier coefficients in Part 1 of this paper is de- generalized to a closed form expression similar to that presented in ref. [13]:

$$
\begin{aligned}
& \theta_{1}=0 \text { and } \theta_{2}=\cos ^{-1}\left(1-\frac{d r}{R}\right) \text { in up milling and } \\
& \theta_{1}=\cos ^{-1}\left(1-\frac{d r}{R}\right) \text { and } \theta_{2}=180 \text { in down milling }
\end{aligned}
$$

Thus, for square end mill, the geometric and boundary functions all have constant values. Substituting these constants, the expression for the Fourier coefficients in Part 1 of this paper is de- generalized to a closed form expression similar to that presented in ref. [13]:

$$
\begin{aligned}
& \mathrm{A}[N k]=\frac{N}{2 \pi} \mathrm{F}_{1}(N k)=\frac{N}{2 \pi} \int_{\beta_{1}}^{\beta_{2}} h^{\prime}(\beta) e^{-j N k \beta} d \beta \sum_{i=1}^{2} \mathbf{q}_{i} \mathbf{P}_{i w}(N k)=\frac{N \cdot H^{\prime}(N k)}{2 \pi} \sum_{i=1}^{2} \mathbf{q}_{i} \mathbf{P}_{i w}(N k) \\
& =\frac{N \cdot H^{\prime}(N k)}{2 \pi}\left(\left[\begin{array}{ccc}
1 & k_{r s} & 0 \\
-k_{r s} & 1 & 0 \\
0 & 0 & -k_{a s}
\end{array}\right] \mathbf{P}_{1 w}(N k)+\left[\begin{array}{ccc}
1 & k_{r p} & 0 \\
-k_{r p} & 1 & 0 \\
0 & 0 & -l k_{a p}
\end{array}\right] \mathbf{P}_{2 w}(N k)\right.
\end{aligned}
$$

where $H(N k)$ is the Fourier transform of the windowed chip width density function, $H^{\prime}(\omega)$, evaluated at $\omega=N k$. The frequency spectra are explicitly determined by the product of $\mathbf{P}_{i w}(N k)$ and $H^{\prime}(N k)$. The values of $\mathbf{P}_{i w}(N k)$ 's are related to the radial depth of cut and have been discussed in Part 1 of this paper. Spectra characteristics of $H^{\prime}(\omega)$ are similar to those of the radial cutting window function, $W_{r}(\omega)$, discussed in Part 1 with periodic zeros at $\omega=2 k \pi / \beta_{2} . \beta_{2}$ is in turn determined by the axial depth of cut through Eq. (1); therefore, the value of $H^{\prime}(\mathrm{Nk})$ can be shown to vanish at axial depths of cut with

$$
d a=\frac{2 m \pi R}{N \cdot \tan \alpha} m=1,2 \ldots
$$

Under these axial depths of cut, the dynamic forces will vanish completely regardless of the values of $\mathbf{P}_{i w}(N k)$ 's and only the average forces remain in the $\mathrm{X}, \mathrm{Y}$ and $\mathrm{Z}$ directions.

\section{Taper end mills}

For a taper end mill shown in Figure 1b, the axial elevation angle $\psi_{0}$ is a constant and the radius of the cutter is a linear function of $h$ :

$$
R(h)=R_{o}+h \cot \psi_{o}
$$

The radial depth of cut for the configuration shown becomes

$$
d r(h)=d r_{o}+h \cot \psi_{o}
$$

The entry and exit angles are therefore no longer constant as in the case of the cylindrical end mill and can be determined from Eq. (2) as a function of $h$. The curvilinear geometry of the cutting edge is generally defined by two types of helical functions: constant helix angle or a constant helical lead.

i) For a taper end mill with a constant helix angle, $\alpha_{0}$, the helix lead is

$h^{\prime}(\beta)=\frac{d h}{d \beta}=\frac{R(\beta)}{\tan \alpha_{o}}$

Substituting Eq. (4) into (6) and considering the boundary condition of $\beta=0$ at $h=0, h(\beta)$ can be shown to be:

$$
h(\beta)=\frac{R o}{\cot \psi_{O}}\left(\exp \left(\frac{\cot \psi_{O}}{\tan \alpha_{O}} \beta\right)-1\right)
$$

Substituting Eq. (6) into Eqs. (3), (4) and (5) results in

$$
\mathrm{R}(\beta)=\mathrm{R}_{\mathrm{o}} \exp \left(\frac{\cot \psi_{\mathrm{o}}}{\tan \alpha_{\mathrm{o}}} \beta\right)
$$

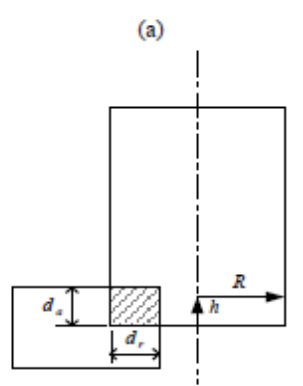

(c)
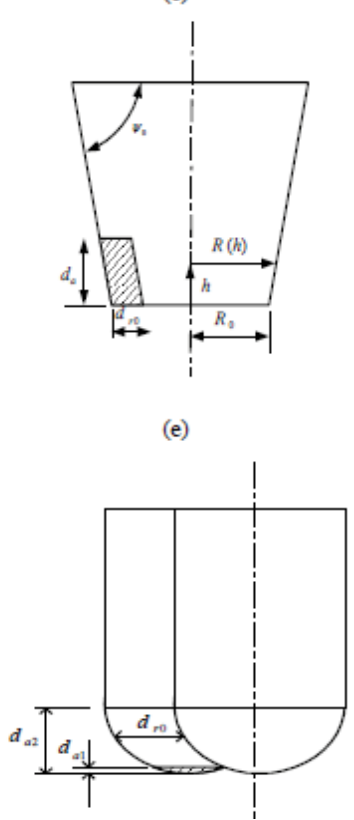

Figure 1: Cutting geometry for (a): a cylindrical end mill, (b) \& (c): a taper end mill, and $(\mathrm{d}) \&(\mathrm{e})$ : a ball end mill. 


$$
d r(\beta)=d r_{0}+R_{0}\left(\exp \left(\frac{\cot \psi_{o}}{\tan \alpha_{o}} \beta\right)-1\right) \text { and } h^{\prime}(\beta)=\frac{R_{0}}{\tan \alpha_{o}} \exp \left(\frac{\cot \psi_{o}}{\tan \alpha_{o}} \beta\right)
$$

From Eqs. (1) and (8), the entry/exit angles along the cutting edge, $\theta_{1}(\beta)$ and $\theta_{2}(\beta)$, can be obtained. The radial range of immersion starts at $\beta_{1}=0$ with $h_{1}=0$ and ends at $\beta_{2}$, which can be found from Eq. (7) to be

$$
\beta_{2}=\frac{\tan \alpha}{\cos \psi_{o}} \ln \left(1+\frac{\mathrm{d}_{\mathrm{a}} \cos \psi_{o}}{R_{0}}\right)
$$

ii) For a taper end mill with a constant helix lead with

$$
h^{\prime}(\beta)=\frac{R_{o}}{\tan \alpha_{0}}
$$

where $\alpha_{o}$ is the nominal helix angle at the bottom of the cutter at $h=0$ with $\beta=0$, its cutting edge is defined by the following constraint equation,

$$
h=\frac{R_{o}}{\tan \alpha_{0}} \beta
$$

The cutter radius as a function of $\beta$ can be found from Eq. (4) to be

$$
\mathrm{R}(\beta)=R_{o}+h \cot \psi_{o}=R_{o}+\frac{R_{o} \cot \psi_{o}}{\tan \alpha_{o}} \beta
$$

For the cutting configuration shown in Figure $2 \mathrm{~b}$, the radial depth of cut as a function of $\beta$ becomes

$$
d r(\beta)=\left(R_{o}-C_{o}\right)+\frac{R_{o} \cot \psi_{o}}{\tan \alpha_{o}} \beta
$$

For a milling process with consecutive passes as shown in Figure $2 c$, the radial side step $d r_{0}$ will be the radial depth of cut for each cutting point so that $d r(\beta)=d r_{0}$. The entry and exit angles as a function of $\beta$ and can be found from Eq. (2) together with Eqs. (12) and (13). The two end points of the radial engagement are

$$
\beta_{1}=0 \text { and } \beta_{2}=\frac{d_{a} \tan \alpha_{0}}{R_{o}}
$$

The required boundary functions and the helix lead for two types of taper end mills has been derived in the above so that Fourier coefficients of the milling forces can be calculated. Although these two types of taper end mills have different mathematical representations, $\beta$ as function of $h$ for both cutters are found to be practically the same for a typical $\alpha_{0}=30^{\circ}$ cutter with $h / R_{0}<1$ as shown in Figure 2 for cutters of four different taper angles. Therefore, the helix lead and the boundary functions can be treated equally for both types of taper end mills and their milling forces can be inferred to be practically the same.

It should be noted that the Fourier coefficients of cutting forces for taper end mills in slot milling or half slot milling can be expressed in a closed form like Eq. (3):

$$
\mathbf{A}[\mathrm{N} k]=\frac{N \cdot H^{\prime}(N k)}{2 \pi}\left(\mathbf{q}_{1}(\psi) \mathbf{P}_{1 \mathrm{w}}(N k)+\mathbf{q}_{2}(\psi) \mathbf{P}_{2 \mathrm{w}}(N k)\right)
$$

Since $H^{\prime}(\omega)$ have periodic zeros at $\omega=2 k \pi / \beta_{2}, H^{\prime}(N k)$ can be shown to vanish at axial depths of cut with

$$
d a_{t 1}=\frac{2 m \pi R_{0}}{N \cdot \tan \alpha_{0}} \quad m=1,2 \ldots \text { (for mill with a constant }
$$

helix lead)

$d a_{t 2}=\frac{R_{o}}{\cot \psi_{o}}\left(\exp \left(\frac{2 m \cot \psi_{o}}{N \cdot \tan \alpha_{o}}\right)-1\right) m=1,2 \ldots$ (for mill with a constant

$$
\text { helix angle) }
$$

which indicates that selecting the axial depths of cut as in (17a) or (17b) in slot milling and half slot milling can only get average, or DC, force component, in which dynamic force components due to shearing and ploughing mechanism will vanish completely regardless of the flute number of taper end mill. However, when $\mathrm{N}<8$ (i.e., $\beta_{2}>^{\pi / 4}$ ), $d a_{t 2}$ is larger than $d a_{t 2}$ under the same $R_{0}$ for $\psi>40^{\circ}$ as shown in Figure 2.

\section{Ball end mills}

The axial cross section profile of the ball-end cutter shown in Figure $1 \mathrm{~d}$ is defined by

$$
d h=R_{0} \sin \psi d \psi
$$

As for the taper end mill, two types of curvilinear edges will be used.

i) A ball-end cutter with constant helix lead will have

$$
h^{\prime}(\beta)=\frac{R_{o}}{\tan \alpha_{0}}
$$

where $R_{o}$ is the ball radius and $\alpha_{0}$ the nominal helix angle at $h=R_{0}$.

Combining Eqs. (15) and (18) with the boundary condition of $\psi=0$ at $\beta=0, \psi$ can be shown to be

$$
\psi=\cos ^{-1}\left(1-\frac{\beta}{\tan \alpha_{0}}\right)
$$

The radius and radial depth of cut for a point at $\beta$ along the helical edge thus can be expressed as

$$
\mathrm{R}(\beta)=R_{o} \sqrt{\frac{\beta}{\tan \alpha_{\theta}}\left(2-\frac{\beta}{\tan \alpha_{\theta}}\right)} \text { and } d r(\beta)=R_{0} \sqrt{\frac{\beta}{\tan \alpha_{0}}\left(2-\frac{\beta}{\tan \alpha_{0}}\right)-\mathrm{c}_{0}}
$$

from which the entry and exit angles as function of $\beta$ can be found. For the configuration shown in Figure 2d for shoulder milling, the end points of the radial engagement are determined by

$$
\left.\beta_{1}=\tan \alpha_{0}\left(1-\sqrt{1-\left(\frac{\mathrm{c}_{0}}{R_{0}}\right.}\right)^{2}\right), \beta_{2}=\frac{\tan \alpha_{0} d_{a}}{R_{0}}
$$

ii) For a ball-end cutter with constant helix angle, $\alpha 1$, its helix lead becomes a variable defined by

$$
h^{\prime}(\beta)=\frac{R(\psi)}{\tan \alpha_{1}}=\frac{R_{o} \sin \psi}{\tan \alpha_{1}}
$$

Combining Eqs. (15) and (22) will have

$$
\frac{d \psi}{d \beta}=\frac{1}{\tan \alpha_{1}}
$$

Given the boundary condition of $\psi=0$ at $\beta=0, \psi$ can be shown to be
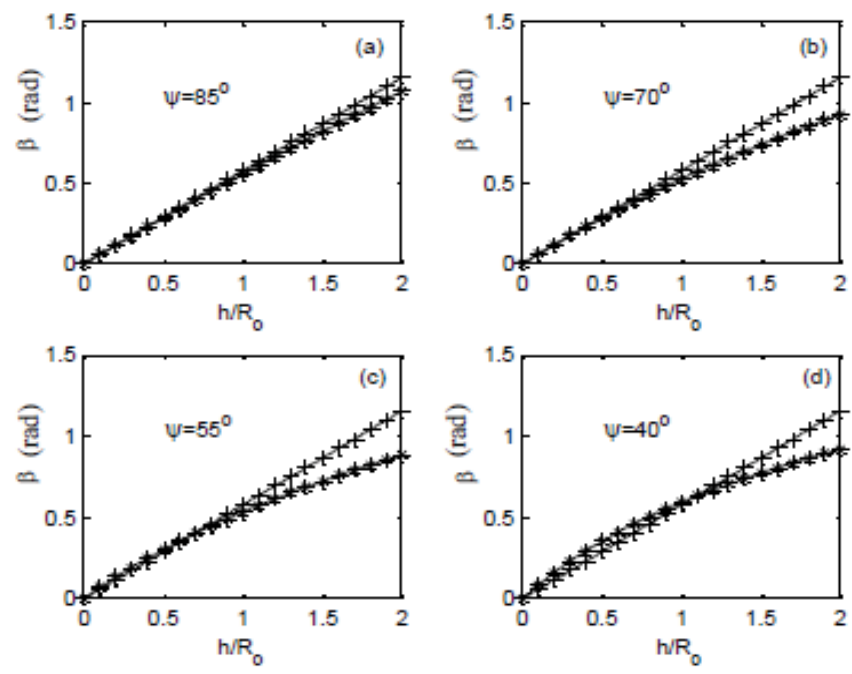

Figure 2: Variation of $\beta$ with respect to cutter height, $h / R o$, for two types of taper end mills; ' + ': constant helix lead and '*': constant helix angle. $\alpha_{0}=\alpha=$ $30^{\circ}$ (a) $\psi=85^{\circ}$, (b) $\psi=70^{\circ}$, (c) $\psi=55^{\circ}$, and (d) $\psi=40^{\circ}$. 


$$
\psi=\frac{\beta}{\tan \alpha_{1}}
$$

The radius and radial depth of cut for a point at $\beta$ along the helical edge thus becomes

$$
R(\beta)=R_{o} \sin \left(\frac{\beta}{\tan \alpha_{1}}\right) \text { and } d r(\beta)=R_{o} \sin \left(\frac{\beta}{\tan \alpha_{1}}\right)-\mathrm{c}_{0}
$$

The integration limits $\beta_{1}$ and $\beta_{2}$ are determined by the upper and lower limits of the axial depth, $d a_{1}$ and $d a_{2}$ by

$$
\beta_{1}=\tan \alpha_{1} \cos ^{-1}\left(1-\frac{d a_{1}}{R_{0}}\right) \text { and } \beta_{2}=\tan \alpha_{1} \cos ^{-1}\left(1-\frac{d a_{2}}{R_{0}}\right)
$$

The major difference between cutters of constant helix lead and constant helix angle is reflected in their $\beta(\psi)$ functions, which can be obtained from Eqs. (20) and (25) as

$$
\beta_{c l}=\tan \alpha_{0}(1-\cos \psi) \text { and } \beta_{c a}=\psi \tan \alpha_{1}
$$

where $\beta_{c l}$ and $\beta_{c a}$ represent the radial angle of the cutter with constant lead and constant helix angle respectively. $\beta_{c l}$ and $\beta_{c a}$ as a function of $\psi$ are plotted in Figure 3 with the assumption of $\alpha_{0}=\alpha_{1}$ $=30^{\circ}$. It is shown that $\beta_{c l}$ and $\beta_{c a}$ are almost in parallel to each other at $\psi>0.57$ or equivalently $h / R_{0}>0.15$. Within this region, $\beta_{c l}$ and $\beta_{c a}$ can be related to each other by the following expression,

$$
\beta_{c l} \approx \beta_{c a}-\left(\frac{\pi}{2}-1\right) \tan \alpha_{1}
$$

at a given $\psi$ or $h$ position. Furthermore, the helix lead $\mathrm{d} h / \mathrm{d} \beta$ can be shown to also be the same.

Assuming the cutting region is not confined to the bottom center, as in the case of Figure 2d, the phase difference in Eq. (29) will be reflected in the phase shift of the total milling forces based on the modified convolution theorem as presented in this study. Given the same cutting conditions and cutting constants for both types of cutters, the Fourier coefficients of the milling forces for the two types of ballend cutters can be shown to have the following relationship:

$$
\mathrm{A}_{\mathrm{cl}}[\mathrm{Nk}]=E X P\left(-j N k\left(\frac{\pi}{2}-1\right) \tan \alpha_{1} \mathrm{~A}_{\mathrm{ch}}[\mathrm{Nk}]\right.
$$

There exists a phase difference of $N k(\pi / 2-1) \tan \alpha_{1}$ for each $k$ th harmonic coefficient while the phase difference is a constant in the angle domain. Figure 4 shows the results of numerical simulation for the two

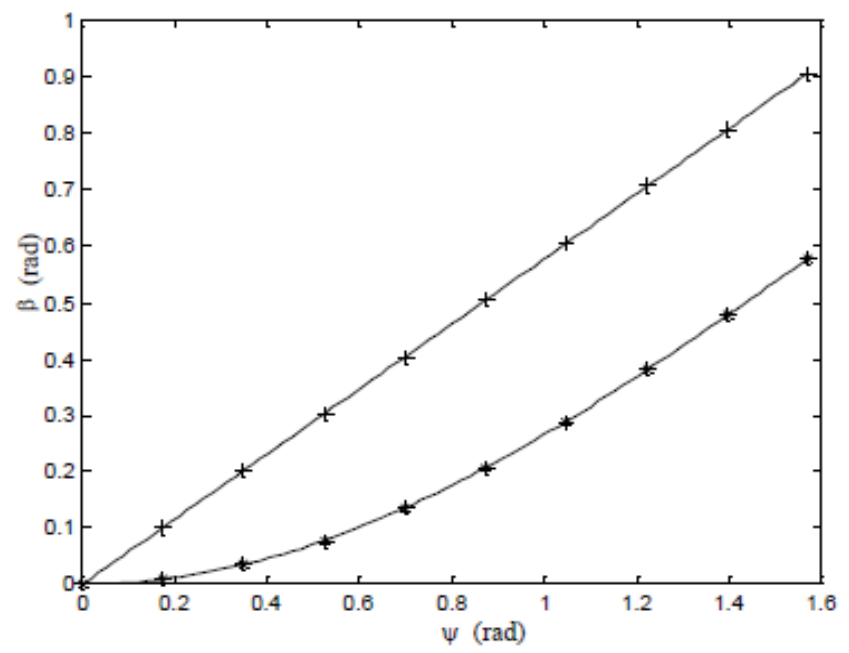

Figure 3: Variation of $\beta$ with respect to the axial elevation angle $\psi$ for two types of ball-end mills; ' + ': constant helix angle '*': constant helix lead. $\alpha_{0}=\alpha=30^{\circ}$.
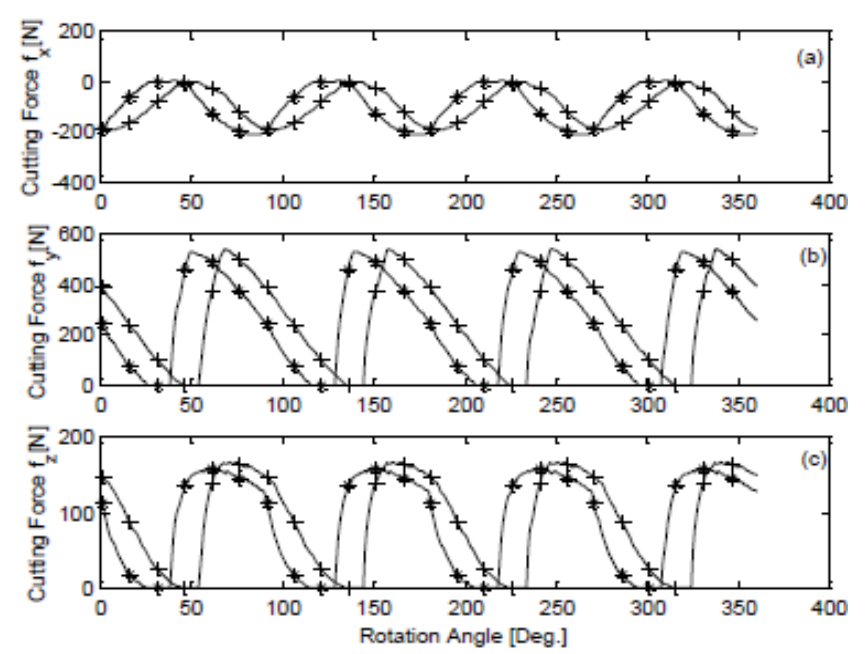

Figure 4: Simulated cutting forces for two types of ball-end mills; '+': constant helix angle and '*': constant helix lead. $\alpha_{0}=\alpha=30^{\circ}, R_{0}=8 \mathrm{~mm}, d a=7 \mathrm{~mm}, c_{0}$ $=3 \mathrm{~mm}, t x=0.07 \mathrm{~mm} /$ tooth, $N=4$, down milling.

types of cutters with $\alpha_{0}=\alpha_{1}=30^{\circ}$ in the shoulder milling configuration as shown in Figure 1d. The phase difference is shown to be about $18^{\circ}$. Figure 1e shows the common step-over milling process for a ball-end mill. For the purpose of finding the milling forces, this milling process can be treated as the combination of a slot milling process and a regular milling process with a constant radial depth of cut. The total milling forces are therefore the sum of forces from these two milling processes:

(a) For $0 \leq h \leq d a_{1}$, this is a slot milling process with the axial depth, $d a_{1}$, determined by the ball radius $R_{0}$ and the side step $d r_{0}$ with

$$
d a_{1}=R_{0}-\sqrt{R_{0}^{2}-\left(\frac{d r_{o}}{2}\right)^{2}}
$$

Within this cutting region, the entry and exit angles are $\theta 1=0$ and $\theta 2=\pi$, and the axial cutting range starts from 0 and ends at $\beta_{2}$, which is determined by $d a 1$ through Eq. (22) or Eq. (27).

(b) For $d a_{1} \leq h \leq d a_{2}$, the entry and exit angles are determined from Eq. (2) by the step size, $d r_{0}$, and $R(\beta)$, which is derived from Eq. (21) or (26). The end points of the radial engagement range are $\beta_{2}$ and $\beta_{3}$, which correspond to the axial depths $d a_{1}$ and $d a_{2}$ according to Eq. (22) or Eq. (27). Geometric functions thus have been derived for cutters of three external profiles and of five different helical geometries. The entry and exit angles of the boundary functions are obtained mostly indirectly by showing the cutter radius and radial depth of cut as a function of $\beta$. Fourier coefficients can be readily evaluated for these cutters and the selected cutting configurations.

Except for the case of the cylindrical end mill, a closed form expression for the Fourier coefficients of the frequency domain forces model cannot be derived owing to the presence of transcendental functions, $\psi(\beta) h^{\prime}(\beta) \mathbf{P}_{\mathrm{iw}}(\omega, \beta)$, in the integrand, and numerical integration will be required for their evaluation.

\section{Average Forces for the General and Common Helical Cutters}

Among the Fourier coefficients for the milling forces, the average forces are the easiest to obtain. They have also been known to have a strong influence on the stability and dimensional error problems of the milling processes. Analytical expressions for the average forces should 
facilitate the analysis and understanding of these problems. In this section, general expressions for the average forces for the helical end mills and specific expressions for three types of cutters will be derived and their characteristics discussed.

A general expression for the average forces of a general helical end mill can be written by setting $\omega=0$ in the Fourier coefficient expression of the milling forces such that:

$$
\mathrm{A}[0]=\frac{N}{2 \pi} \sum_{i=1}^{2} \int_{\beta_{1}}^{\beta_{2}} \mathbf{q}_{i}(\beta) h^{\prime}(\beta) \mathbf{P}_{\mathrm{iw}}(0, \beta) d \beta
$$

Where

$$
\mathbf{P}_{1 \mathrm{w}}(0, \beta)=\left(\begin{array}{l}
-\frac{1}{4} \cos 2 \theta \\
\frac{1}{2}\left(\theta-\frac{1}{2} \sin 2 \theta\right) \\
-\cos \theta
\end{array}\right)_{\theta=q(\beta)}^{\theta_{2}(\beta)} \text { and } \mathbf{P}_{2 \mathrm{~W}}(0, \beta)=k_{t p}\left(\begin{array}{l}
\sin \theta \\
-\cos \theta \\
\theta
\end{array}\right)_{\theta=\theta_{(\alpha)}(\beta)}^{\theta_{2}(\beta)}
$$

Eq. (32) shows that the average forces are proportional to the flute number and are physically the sum of the local average forces contributed by all cutting points along the cutting edge. Eqs. (32) and (33) are applicable for all types of helical cutters. Eq. (33) can be simplified for the common up and down cut configurations. For up milling, $\theta_{1}=0$ and Eq. (33) can be reduced to

$$
\mathbf{P}_{1 \mathrm{w}}(0, \beta)=k_{t s} t_{x}\left(\begin{array}{l}
\frac{1}{4}\left(1-\cos 2 \theta_{2}\right) \\
\frac{1}{2}\left(\theta_{2}-\frac{1}{2} \sin 2 \theta_{2}\right) \\
1-\cos \theta_{2}
\end{array}\right) \text { and } \mathbf{P}_{2 \mathrm{w}}(0, \beta)=k_{t s} t_{x}\left(\begin{array}{l}
\sin \theta_{2} \\
1-\cos \theta_{2} \\
\theta_{2}
\end{array}\right)
$$

And for down milling, $\theta_{2}=\pi$, Eq. (33) becomes

$$
\mathbf{P}_{1 \mathrm{w}}(0, \beta)=k_{t s} t_{x}\left(\begin{array}{l}
\frac{1}{4}\left(\cos 2 \theta_{1}-1\right) \\
\frac{1}{2}\left(\pi-\theta_{1}\right)+\frac{1}{4} \sin \theta_{1} \\
1+\cos \theta_{1}
\end{array}\right) \text { and } \mathbf{P}_{2 \mathrm{w}}(0, \beta)=k_{t s} t_{x}\left(\begin{array}{l}
-\sin \theta_{1} \\
1+\cos \theta_{1} \\
\pi-\theta_{1}
\end{array}\right)
$$

With the geometric functions and the boundary functions derived in the previous sections, the average forces in Eq. (32) can always be obtained by the numerical integration presented in part 1 . However, simpler forms of expressions for the average forces can be derived under some special cutting conditions and their evaluation can be simplified.

For a cylindrical end mill as shown in Figure 1a, Eq. (32) can be reduced to the following closed form expression:

$$
\mathrm{A}[0]=\frac{N d_{a}}{2 \pi}\left(\left[\begin{array}{ccc}
1 & k_{r s} & 0 \\
-k_{r s} & 1 & 0 \\
0 & 0 & -k_{r s}
\end{array}\right] \mathbf{P}_{1 \mathrm{~W}}(0)+\left[\begin{array}{ccc}
1 & k_{r p} & 0 \\
-k_{r p} & 1 & 0 \\
0 & 0 & -k_{r p}
\end{array}\right] \mathbf{P}_{2 \mathrm{w}}(0)\right)
$$

For the taper end mill with constant helix angle, Eq. (32) is reduced to

$$
\mathrm{A}[0]=\frac{N}{2 \pi} \frac{R_{o}}{\tan \alpha_{0}} \sum_{i=1}^{2} \mathbf{q}_{i}\left(\psi_{o}\right) \int_{0}^{\beta_{a}} \exp \left(\frac{\cot \psi_{o}}{N \cdot \tan \alpha_{0}}\right) \mathbf{P}_{\mathrm{iw}}(0, \beta) d \beta
$$

and for the taper end mill with constant helix lead, Eq. (32) becomes

$$
\mathrm{A}[0]=\frac{N}{2 \pi} \frac{R_{o}}{\tan \alpha_{0}} \sum_{i=1}^{2} \mathbf{q}_{i}\left(\psi_{o}\right) \int_{0}^{\beta_{a}} \mathbf{P}_{\mathrm{iw}}(0, \beta) d \beta
$$

The evaluation of average forces still requires numerical integration for most cutting configurations except for the following special cutting conditions.
In the special cases of half slot and slot milling conditions a single closed form expression for the average forces can be obtained for all types of helical cutters. For the slot milling process, $\theta_{1}=0$ and $\theta_{2}=\pi$, the two vector functions in Eq. (33) are reduced to constant values with

For half slot milling, these two vectors also have constant values. For up cut configuration, they are

$$
\mathbf{P}_{1 \mathrm{w}}(0)=k_{t s} t_{x}\left(\begin{array}{l}
P_{1}(0) \\
P_{2}(0) \\
P_{3}(0)
\end{array}\right)=k_{t s} t_{x}\left(\begin{array}{l}
0.5 \\
0.25 \pi \\
1
\end{array}\right)
$$

and

$$
\mathbf{P}_{2 \mathrm{w}}(0)=k_{t p}\left(\begin{array}{l}
P_{4}(0) \\
P_{5}(0) \\
P_{6}(0)
\end{array}\right)=k_{t p}\left(\begin{array}{l}
1 \\
1 \\
0.5 \pi
\end{array}\right)
$$

and for down cut,

$$
\mathbf{P}_{1 \mathrm{~W}}(0)=k_{t s} t_{x}\left(\begin{array}{l}
P_{1}(0) \\
P_{2}(0) \\
P_{3}(0)
\end{array}\right)=k_{t s} t_{x}\left(\begin{array}{l}
-0.5 \\
0.25 \pi \\
1
\end{array}\right)
$$

and

$$
\mathbf{P}_{2 \mathrm{w}}(0)=k_{t p}\left(\begin{array}{l}
P_{4}(0) \\
P_{5}(0) \\
P_{6}(0)
\end{array}\right)=k_{t p}\left(\begin{array}{l}
-1 \\
1 \\
0.5 \pi
\end{array}\right)
$$

Since these $\mathbf{P}_{\mathrm{i}}(0)$ 's are constant, Eq. (32) becomes an analytically integrable form for the slot and half slot milling conditions:

$$
\mathrm{A}[0]=\frac{N}{2 \pi} \sum_{i=1}^{2}\left(\int_{0}^{\beta_{a}} \mathbf{q}_{i}(\beta) h^{\prime}(\beta) d \beta\right) \mathbf{P}_{i}(0)=\frac{N}{2 \pi} \sum_{i=1}^{2}\left(\int_{0}^{d_{a}} \mathbf{q}_{i}(\psi) d h\right) \mathbf{P}_{i}(0)=\frac{N}{2 \pi} \sum_{i=1}^{2} \mathbf{Q}_{i} \mathbf{P}_{i}(0)
$$

where $\mathbf{Q}_{i}$ can be shown to be

$$
\begin{aligned}
& \mathbf{Q}_{1}=R_{o}\left[\begin{array}{ccc}
Q_{1} & k_{r s} Q_{3}+k_{a s} Q_{2} & 0 \\
-\left(k_{r s} Q_{3}+k_{a s} Q_{2}\right) & Q_{1} & 0 \\
0 & 0 & k_{r s} Q_{2}-k_{a s} Q_{3}
\end{array}\right] \\
& \mathbf{Q}_{2}=R_{o}\left[\begin{array}{ccc}
Q_{5} & k_{r p} Q_{6}+k_{a p} Q_{4} & 0 \\
-\left(k_{r p} Q_{6}+k_{a p} Q_{4}\right) & Q_{5} & 0 \\
0 & 0 & k_{r p} Q_{4}-k_{a p} Q_{6}
\end{array}\right]
\end{aligned}
$$

In which

$$
\begin{aligned}
& Q_{1}=\frac{1}{R_{o}} \int_{0}^{d_{a}} d h=\frac{d_{a}}{R_{o}}, Q_{2}=\frac{1}{R_{o}} \int_{0}^{d_{a}} \cos \psi d h=\frac{1}{R_{o}}=\frac{1}{R_{o}} \int_{0}^{\psi_{a}} \cos \psi \frac{d h}{d \psi} d \psi \\
& Q_{3}=\frac{1}{R_{o}} \int_{0}^{d_{a}} \sin \psi d h=\frac{1}{R_{o}} \int_{0}^{\psi_{a}} \sin \psi \frac{d h}{d \psi} d \psi, Q_{4}=\frac{1}{R_{o}} \int_{0}^{d_{a}} \cot \psi d h=\frac{1}{R_{o}} \int_{0}^{\psi_{a}} \cot \psi \frac{d h}{d \psi} d \psi \\
& Q_{5}=\frac{1}{R_{o}} \int_{0}^{d_{a}} \csc \psi d h=\frac{1}{R_{o}} \int_{0}^{\psi_{a}} \csc \psi \frac{d h}{d \psi} d \psi, Q_{6}=\frac{1}{R_{o}} \int_{0}^{d_{a}} d h=\frac{d_{a}}{R_{o}}
\end{aligned}
$$

The axial immersion angle, $\psi_{a}$, in Eq. (45) for the ball-end cutter can be found directly from Eq. (20) or (25). Values of $Q_{i}$ 's for the square, taper, and ball end mills are listed in Table 1.

Eqs. (42-45) for the average forces in the slot or half slot milling conditions are applicable not only for the three types of cutters discussed here, but also for all helical cutters of different external profile and curvilinear geometry. Assuming the cutting constants are the same, it is therefore shown through these equation that the average forces in slot or half slot milling are independent of their helical lead, $h^{\prime}(\beta)$, and are only dependent on the external profile of the cutter through function $h(\psi)$ in Eq. (45). 


\section{Identification of the Shearing and Ploughing Cutting Constants}

For all types of milling force models, both in the numerical or analytical form and in the angle or frequency domain, the accuracy of the force prediction bears on the trueness of the cutting coefficients. However, cutting constants are difficult to predict and are mostly taken or calculated from a pre-established database through elaborative cutting tests. The analytical nature of the presented frequency domain milling force model allows the direct identification of the six unknown cutting constants from the measured milling forces. With the least signal processing and mathematical complexity required as well as considering the simplicity for the test set up, the cutting constants can be identified using the closed-form expression of Eq. (42) for the average cutting forces in the slot or half slot milling operation. Eq. (42) can be rearranged as the following linear equation in the unknown shearing and ploughing cutting constants:

$$
\begin{aligned}
& {\left[\begin{array}{l}
A_{x}[0] \\
A_{y}[0] \\
A_{z}[0]
\end{array}\right]=\left[\begin{array}{l}
A_{x}[0] \\
A_{y}[0] \\
A_{z}[0]
\end{array}\right]_{\text {shearing }}+\left[\begin{array}{l}
A_{x}[0] \\
A_{y}[0] \\
A_{z}[0]
\end{array}\right]_{\text {ploughing }}=\frac{N R_{o} t_{x}}{2 \pi} \mathbf{T}_{s} \mathbf{k}_{s}+\frac{N R_{o}}{2 \pi} \mathbf{T}_{\mathbf{p}} \mathbf{k}_{\mathbf{p}}} \\
& \text { where } \mathbf{T}_{s}=\left[\begin{array}{ccc}
Q_{1} P_{1}(0) & Q_{3} P_{2}(0) & Q_{2} P_{2}(0) \\
Q_{1} P_{2}(0) & -Q_{3} P_{1}(0) & -Q_{2} P_{1}(0) \\
0 & Q_{2} P_{3}(0) & -Q_{3} P_{3}(0)
\end{array}\right] ; \mathbf{k}_{s}=\left[\begin{array}{l}
k_{t s} \\
k_{r s} k_{t s} \\
k_{a s} k_{t s}
\end{array}\right] \\
& \text { and } \mathbf{T}_{\mathbf{p}}=\left[\begin{array}{ccc}
Q_{5} P_{4}(0) & Q_{6} P_{5}(0) & Q_{4} P_{5}(0) \\
Q_{5} P_{5}(0) & -Q_{6} P_{4}(0) & -Q_{4} P_{4}(0) \\
0 & Q_{4} P_{6}(0) & -Q_{6} P_{6}(0)
\end{array}\right] ; \mathbf{k}_{\mathbf{p}}=\left[\begin{array}{l}
k_{t s} \\
k_{r p} k_{t p} \\
k_{a p} k_{t p}
\end{array}\right]
\end{aligned}
$$

Three equations for the three average force components can be obtained from each cutting test. Therefore, two sets of measured average cutting forces data with different cutting conditions are required to make up the system of equations in solving the six cutting constants. By regrouping the matrix equation in (46), a closed form formula for the identification of the six cutting constants can be written for a general helical end mill as follows:

$$
\left[\begin{array}{l}
k_{t s} \\
k_{r s} k_{t s} \\
k_{a s} k_{t s} \\
k_{t p}
\end{array}\right]=\frac{2 \pi}{N R_{o}} \mathbf{T}^{-1}\left[\begin{array}{l}
A_{x 1}[0] \\
A_{y 1}[0] \\
A_{z 1}[0] \\
A_{x 2}[0] \\
A_{y 2}[0] \\
A_{z 2}[0]
\end{array}\right] \text { where } \mathbf{T}=\left[\begin{array}{ll}
t_{x 1} \mathbf{T}_{s 1} & \mathbf{T}_{\mathbf{p} 1} \\
t_{x 2} \mathbf{T}_{s 2} & \mathbf{p}_{\mathbf{p} 1}
\end{array}\right]
$$

and subscripts ' 1 ', ' 2 ' indicate the two different cutting tests.

Both the cutting tests and the computations can be further simplified if the same cutting conditions, except the feed speed are chosen for these two slot or half slot milling processes. In that case, the T matrix in Eq. (49) becomes

$$
\mathbf{T}=\left[\begin{array}{ll}
t_{x 1} \mathbf{T}_{s} & \mathbf{T}_{\mathbf{p}} \\
t_{x 2} \mathbf{T}_{s} & \mathbf{T}_{\mathbf{p}}
\end{array}\right]
$$

Eq. (49) or Eq. (50) has provided a convenient formula for the identification of six shearing and ploughing cutting constants for all types of helical cutters through two cutting tests with the slot or half slot milling condition.

\section{Experimental Validation}

Milling experiments were carried out to verify the frequency domain force model and the identification formula for the cutting constants. The cutting forces were measured with the Kistler 9255B dynamometer. Three different work/cutter pairs are used: a tapered end mill with AL7075-T6, a ball-end mill with AL2024-T4 and a cylindrical end mill with AL2024-T4. Typical yield and tensile strengths are 503
$\mathrm{MPa}$ and $572 \mathrm{MPa}$ respectively for 7075-T6, and $325 \mathrm{MPa}$ and 470 $\mathrm{MPa}$ for AL2024-T4. With average uncut chip thickness ranging from 0.02 to $0.1 \mathrm{~mm}$, five sets of cutting constants are identified from 10 slot milling tests for each type of cutter and are shown with respect to the
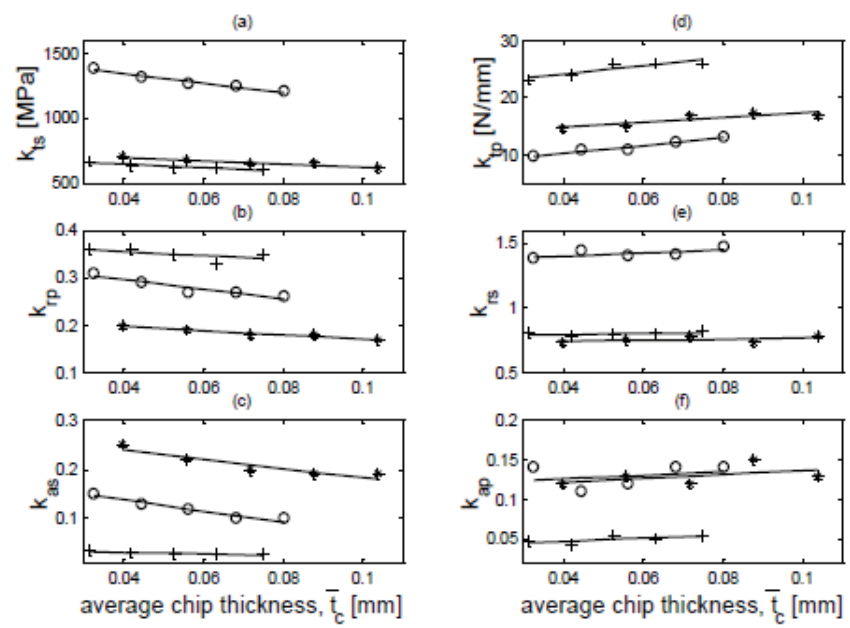

Figure 5: The identified cutting constants vs. average chip thickness. (a), (b), and (c) are for the tangential, radial, and axial shearing constants. (d), (e), and (f) are for the tangential, radial, and axial ploughing constants. 'o': ball end mill, '+': taper end mill, and '*': square end mill.

\begin{tabular}{|c|c|c|c|c|c|c|}
\hline \multirow{2}{*}{ Cutter type } & \multicolumn{3}{|c|}{ Coefficients due to shearing } & \multicolumn{3}{c|}{$\begin{array}{c}\text { Coefficients due to } \\
\text { ploughing }\end{array}$} \\
\cline { 2 - 7 } & $Q_{1}(0)$ & $Q_{2}(0)$ & $Q_{3}(0)$ & $Q_{4}(0)$ & $Q_{5}(0)$ & $Q_{6}(0)$ \\
\hline $\begin{array}{c}\text { Cylindrical } \\
\text { end mill }\end{array}$ & $\bar{d}_{a}$ & 0 & $\bar{d}_{a}$ & 0 & $\bar{d}_{a}$ & $\bar{d}_{a}$ \\
\hline Taper end mill & $\bar{d}_{a}$ & $\bar{d}_{a} \cos \psi_{0}$ & $\bar{d}_{a} \sin \psi_{0}$ & $\bar{d}_{a} \cot \psi_{0}$ & $\bar{d}_{a} \csc \psi_{0}$ & $\bar{d}_{a}$ \\
\hline Ball end mill & $\bar{d}_{a}$ & $\frac{\sin ^{2} \psi_{a}}{2}$ & $\frac{1}{2}\left(\psi_{a}-\frac{\sin 2 \psi_{a}}{2}\right)$ & $\sin \psi_{a}$ & $\bar{d}_{a}$ & $\bar{d}_{a}$ \\
\hline
\end{tabular}

Table 1: Directional coefficients for the average cutting forces in slot milling $\bar{d}_{a}=d_{a} / R_{0}$

\begin{tabular}{|c|c|c|c|c|}
\hline No. & $\mathbf{d a}(\mathbf{m m})$ & $\mathbf{t x} \mathbf{1}(\mathbf{m m} /$ tooth) & $\mathbf{t x 2} \mathbf{( m m / t o o t h )}$ & $\mathbf{t}(\mathbf{m m})$ \\
\hline 1 & 4 & 0.0563 & 0.0688 & 0.0398 \\
\hline 2 & 4 & 0.0812 & 0.0928 & 0.0557 \\
\hline 3 & 4 & 0.106 & 0.119 & 0.0716 \\
\hline 4 & 4 & 0.131 & 0.144 & 0.0875 \\
\hline 5 & 4 & 0.156 & 0.169 & 0.1035 \\
\hline 6 & 6 & 0.0469 & 0.0563 & 0.0327 \\
\hline 7 & 6 & 0.0656 & 0.075 & 0.0446 \\
\hline 8 & 6 & 0.0844 & 0.0938 & 0.0565 \\
\hline 9 & 6 & 0.103 & 0.113 & 0.0684 \\
\hline 10 & 6 & 0.122 & 0.131 & 0.0803 \\
\hline 11 & 3 & 0.0668 & 0.0843 & 0.0315 \\
\hline 12 & 3 & 0.0938 & 0.106 & 0.0421 \\
\hline 13 & 3 & 0.119 & 0.131 & 0.0526 \\
\hline 14 & 3 & 0.144 & 0.156 & 0.0631 \\
\hline 15 & 3 & 0.169 & 0.188 & 0.0749 \\
\hline
\end{tabular}

Table 2: Cutting conditions for the identification of cutting constants. Spindle speed $=400 \mathrm{rpm}$, dry slot cut. No.1-5 are the cylindrical end mill with $N=2, R=5 \mathrm{~mm}$, a $=30^{\circ}$; No.6-10 are for the taper end mil with $N=4, R=5 \mathrm{~mm}, \alpha=30^{\circ}, \psi_{0}=85^{\circ}$; No.10-15 are for the ball end mill with $N=2, R_{o}=5 \mathrm{~mm}, \alpha_{o}=30^{\circ}$. 
Citation: Zheng CM, Junz Wang JJ (2016) Fourier Analysis of Milling Force for General Helical Cutters via Space-time Convolution, Part 2: Applications for Common Cutters and Model Validation. J Appl Mech Eng 5: 195. doi:10.4172/2168-9873.1000195

\begin{tabular}{|c|c|c|c|c|c|c|}
\hline \multirow{2}{*}{$\begin{array}{c}\text { Cutter / work } \\
\text { material }\end{array}$} & \multicolumn{3}{|c|}{ Shearing constants } & \multicolumn{3}{c|}{ Ploughing constants } \\
\cline { 2 - 7 } & $k t s$ (MPa) & krs & kas & $k t p(\mathrm{~N} / \mathrm{mm})$ & krp & $k a p$ \\
\hline $\begin{array}{c}\text { Taper end mill/ } \\
\text { AL7075-T6 }\end{array}$ & 1285 & 0.28 & 0.12 & 11.3 & 1.42 & 0.13 \\
\hline $\begin{array}{c}\text { Cylindrical end mill/ } \\
\text { AL2024-T4 }\end{array}$ & 660 & 0.18 & 0.21 & 16.1 & 0.76 & 0.13 \\
\hline $\begin{array}{c}\text { Ball end mill/ } \\
\text { AL2024-T4 }\end{array}$ & 631 & 0.35 & 0.03 & 25 & 0.8 & 0.05 \\
\hline
\end{tabular}

Table 3: Averages of identified cutting constants. average chip thickness in Figure 5. The three shearing related constants in Figure 5a-c for each work/material pair are shown to increase slightly with decreasing chip thickness, which could be possibly explained by the size effect. The power related tangential shearing constant $k t s$ is the most significant contributor of all to the milling forces. The tangential shearing constant of the AL7075-T6 with the taper end mill is almost twice that of the AL2024-T4 with the ball and cylindrical end mills. The greater difference in the shearing cutting constant seems to reflect more than the mechanical strength of the work material and could be
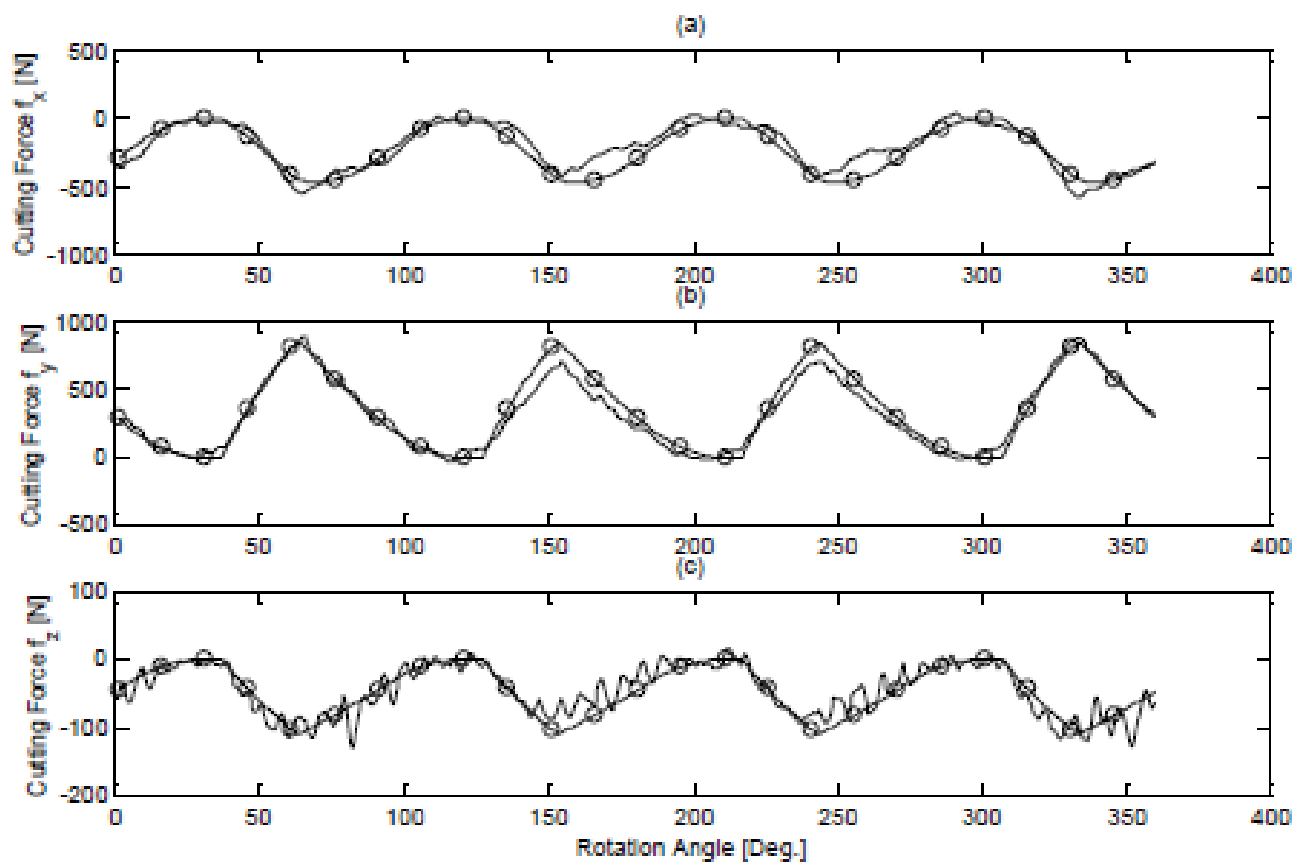

(d)
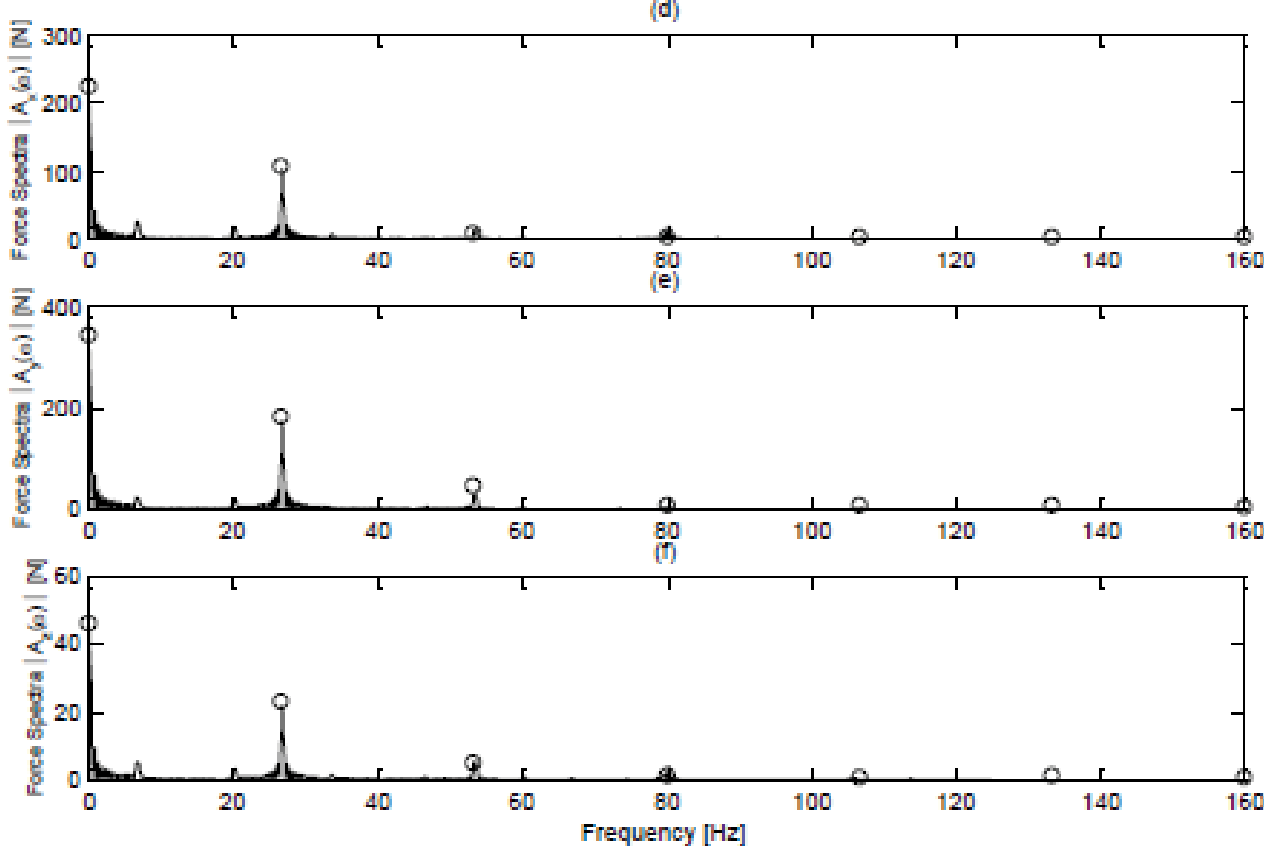

Figure 6: Predicted and measured forces for a taper end mill. Work material: AL7075-T6. $N=4, R_{0}=5 \mathrm{~mm}, \Psi_{\circ}=85^{\circ}, \alpha=30^{\circ}, d a=8 \mathrm{~mm}, d r_{0}=4 \mathrm{~mm}, t x=0.125$ mm/tooth, 400 rpm, dry cut. '-': measured, 'o': predicted. 

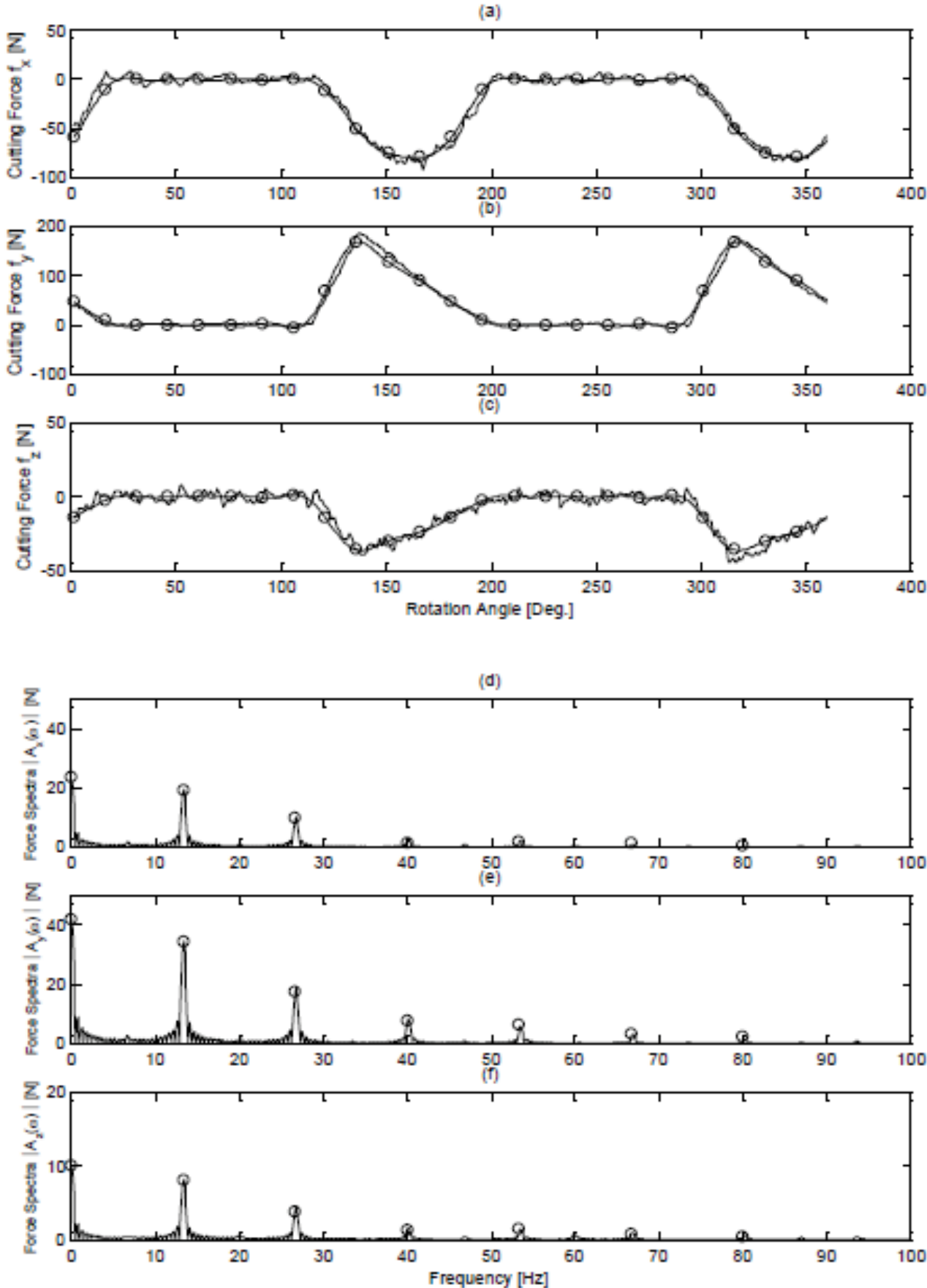

Figure 7: Predicted and measured forces for a cylindrical end mill. Work material: AL2024-T4. $N=2, R o=5 \mathrm{~mm}, d a=3 \mathrm{~mm}, d r=3 \mathrm{~mm}, t x=0.075 \mathrm{~mm} / \mathrm{tooth}$, $N=4$ flutes, $\alpha=30^{\circ}, 400$ rpm, dry cut. '-': measured, 'o': predicted.

partly attributed to the worn tool edge of the taper end mill. The ball and square end mills are both new cutters while the taper end mill is in a used condition. All three ploughing constants for three cutters also remain relatively flat, even showing some reverse trend, Figure $5 \mathrm{~d}-\mathrm{f}$. This slight decrease of ploughing constants with decreasing chip thickness might be explained by the smaller tool edge deformation thus less flank contact area due to the smaller chip load. Compared with the shearing constant, the tangential ploughing constant has a relative small value. However, the ploughing force will be the same as the shearing force for chip thickness in the range of 0.01 to $0.03 \mathrm{~mm}$, which is well within the range encountered in finish milling. As the feed per tooth and the radial depth of cut get smaller such as in high speed milling, the ploughing force will become more significant and its stronger presence should warrant closer examination of its effect on the milling process.

From these identified cutting constants, it is reasonable to use the average cutting constants for cutting force predictions without 

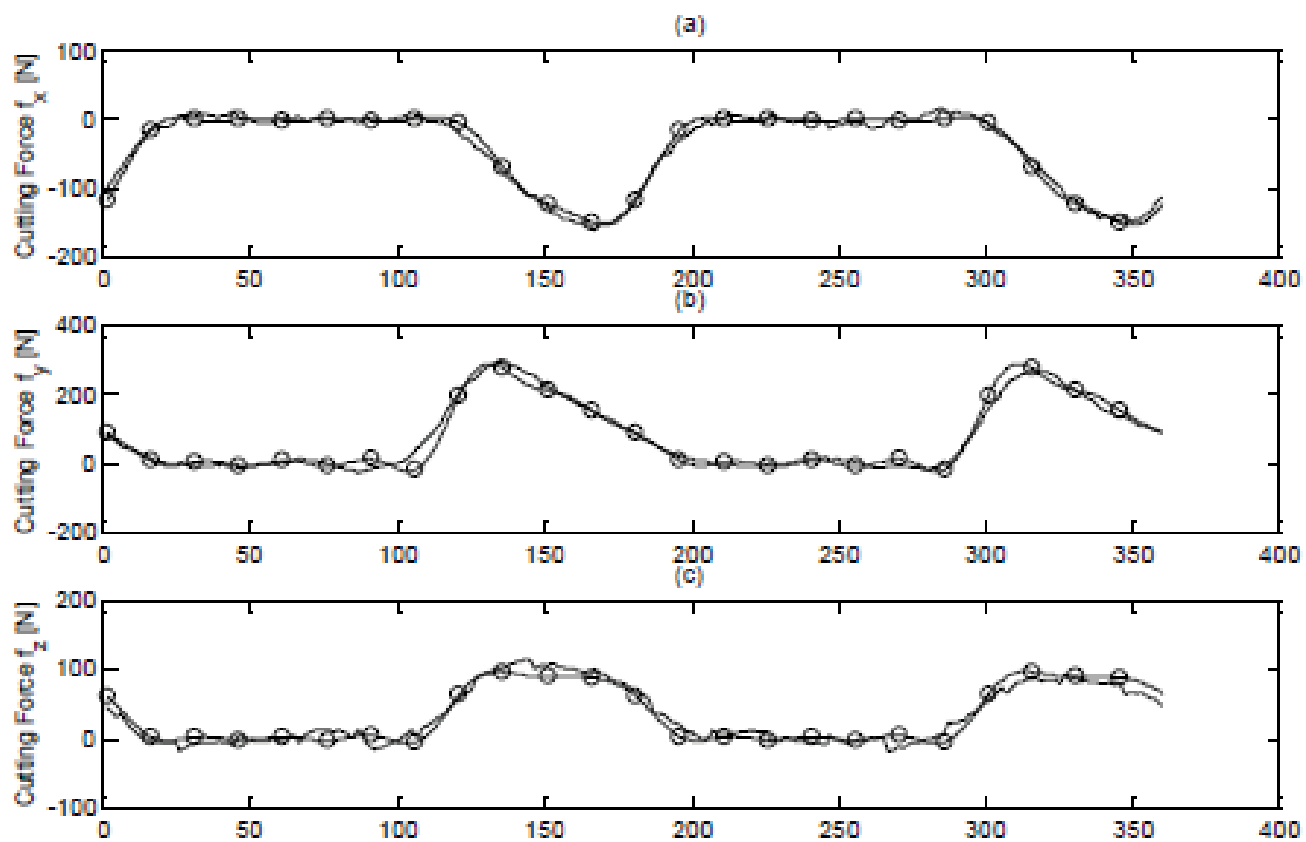

(d)
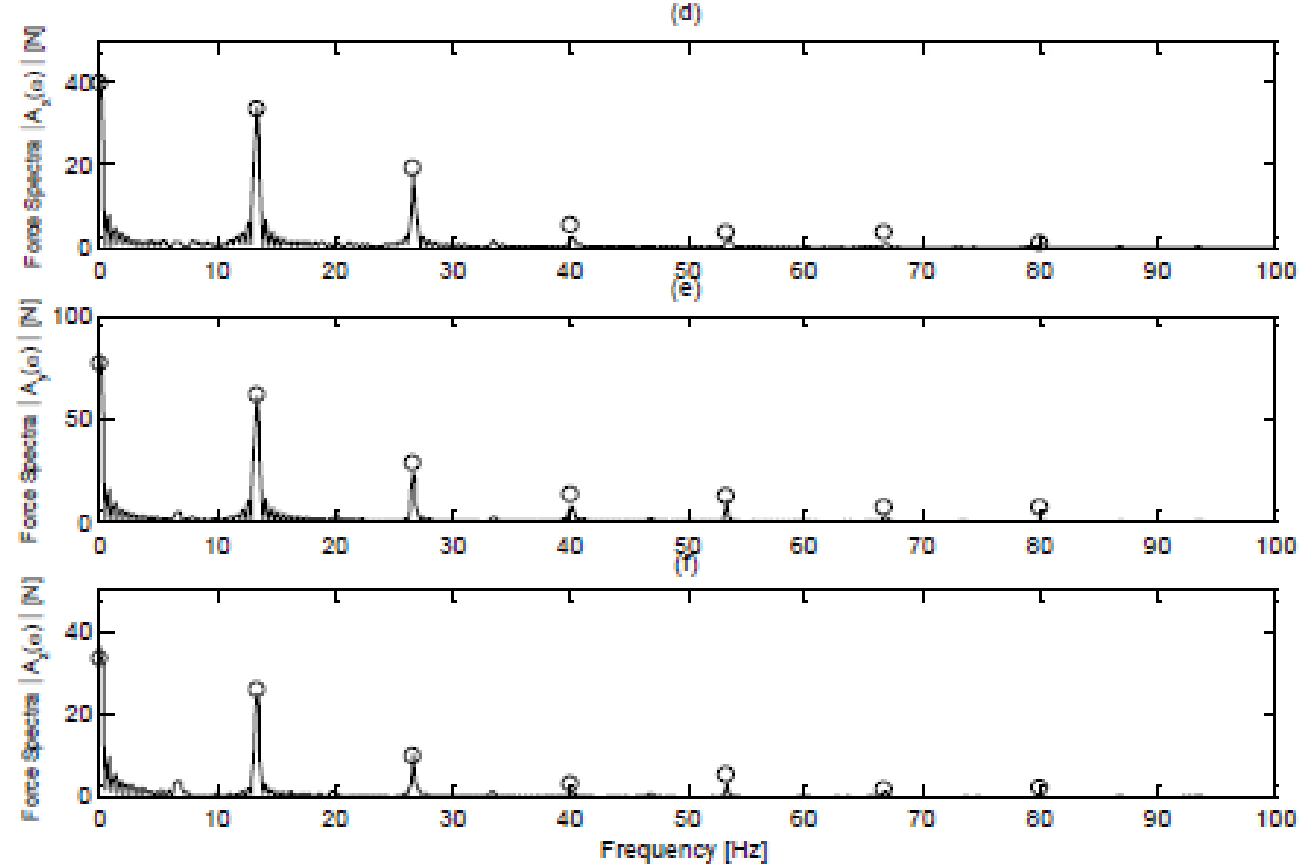

Figure 8: Predicted and measured forces for a ball end mill. Work material: AL2024-T4. $N=2, R_{0}=5 \mathrm{~mm}, d a=3 \mathrm{~mm}, c_{0}=1 \mathrm{~mm}, t x=0.1 \mathrm{~mm} / \mathrm{tooth} \mathrm{a}_{0}=30^{\circ}$, 400 rpm, dry cut. '-': measured, 'o': predicted.

incurring significant error. The average cutting constants for different cutters are listed in Tables 2 and 3 and are used to predict the milling forces for additional cutting tests with different cutting conditions and configurations. The predicted cutting forces are calculated by first finding the Fourier coefficients up to the sixth harmonics by numerical integration with QUAD8 function in Matlab. The angle domain forces are subsequently obtained through Fourier series expansion using inverse Fourier transform function in Matlab. The predicted and experimentally measured milling forces are shown to coincide very well in both the angular and frequency domain as illustrated in Figure 6-8. The Frequency spectra of these milling forces are shown to decrease rapidly as predicted and assume significant magnitude only up to the third harmonics. Sine the Fourier coefficients $\mathbf{A}[N k]$ and $\mathbf{A}[-N k]$ are complex conjugates, only four numerical values for $k$ from 0 to 3 for each force component would be adequate in representing the periodic milling forces.

The forward application of the force model in predicting forces and the inverse application for the identification of cutting constants thus 
proves the validity of the presented frequency domain force model for a generalized helical end mill. Not only are the composing structures and the derived force expressions verified, the basic assumption of constant cutting coefficients with the dual cutting mechanisms for the local forces are also found to be acceptable for the milling process. The fact that these cutting coefficients can be treated as constants within a reasonable range of chip thickness is of great significance in validating the presented model as a linear force model, thus facilitating its further application in the analysis of milling processes.

\section{Conclusions}

Specific applications of the frequency domain force model are illustrated for three common cutters. Geometric functions for the cutter profile, helix lead and entry/exit angles are derived for the square, taper and ball end mills so that numerical values of the force spectra can be calculated for these cutters. For both taper and ball-end cutters, geometric functions for the cutters with a constant helix lead and constant helix angle are separately derived. Assuming the cutting constants are the same, the forces for these two types of taper end mills are shown to have little difference when the axial depth of cut is smaller than the bottom radius. The axial depths of cut can be selected to reduce the force pulsation for the square end mill in peripheral milling and taper end mill in slot and half slot milling. The chosen depth of cut for taper end mill with a constant helix angle is larger than that with a constant helix lead under the same bottom radius when $N<8$ and $\psi$ $>40^{\circ}$.

For the ball-end mills, different helical geometry is shown to only result in a predictable phase difference in the milling forces when the range of axial depth of the cut is not constrained within the bottom two tenths of the ball radius.

A general expression for the average forces of common cutting configurations has been derived for all types of helical cutters. The average forces for slot and half slot milling conditions are presented in a simple closed-form expression and are shown to only be dependent on the external profile of the helical cutter. From this expression for the average forces, an inverse application of the analytical force model is demonstrated to identify the unknown cutting constants from the measured average forces. Milling experiments for each type of cutter were carried out to verify the predictive accuracy of the frequency domain force model and the effectiveness of the identification formula for the six cutting constants.
Based on its analytical nature, this convolution force model can be further explored for other potential applications including the monitoring and identification of other process parameters and using the model as a tool in the design of special helical cutter for the desired force characteristics.

\section{Acknowledgement}

The authors gratefully acknowledge the financial support from Quanzhou Institute of Information Engineering.

\section{References}

1. Martellotti ME (1941) An Analysis of the Milling Process. Transaction of ASME 63: $677-700$.

2. Martellotti ME (1945) An Analysis of the Milling Process Part 2: Down Milling Transaction of ASME 67: 233-251.

3. Yang M, Park H (1991) The Prediction of Cutting Force in Ball End Milling International Journal of Machine Tools \& Manufacture 31: 45-54.

4. Feng $H$, Menq $C$ (1994) The prediction of cutting force in ball-end milling process-Part I: Model formulation and model building procedure. Int $\mathrm{J}$ of Machine Tool Design and Manufacture 34: 697-710.

5. Feng H, Menq C (1994) The prediction of cutting force in ball-end milling process-Part II: Cut Geometry Analysis and Model Verification. Internationa Journal of Machine Tools \& Manufacture 34: 711-719.

6. Yucesan G, Altintas $Y$ (1996) Prediction of ball end milling forces. ASME Journal of Engineering for Industry 118: 95-103.

7. Imani BM, Sadeghi MH, Elbestawi MA (1998) An improved process simulation system for ball-end milling of sculptured surfaces. International Journal of Machine Tools \& Manufacture 38: 1089-1107.

8. Sonawane HA, Joshi SS (2010) Analytical modeling of chip geometry and cutting forces in helical ball end milling of superalloy Inconel 718. CIRP Journal of Manufacturing Science and Technology 3: 204-217.

9. Ramaraj TC, Eleftheriou E (1994) Analysis of the mechanics of machining with tapered end-milling cutters. ASME Journal of Engineering for Industry 116: 398-404.

10. Huang T, Whitehouse DJ (1999) Cutting force formulation of taper end-mills using differential geometry. Precision Engineering 23: 196-203.

11. Altintas Y, Lee P (1996)A General Mechanics and Dynamics Model for Helical End Mills. Annals of CIRP 45: 59-64.

12. Engin S, Altintas $Y$ (2001) Mechanics and dynamics of general milling cutters Part I: helical end mills. International Journal of Machine Tools \& Manufacture 41: 2195-2212.

13. Wang JJ, Zheng CM (2002) An analytical force model with shearing and ploughing mechanisms for end milling. International Journal of Machine Tools \& Manufacture 42: 761-771. 